\title{
SENTIDOS Y SIGNIFICADOS SOBRE LA EDUCACIÓN Y EL TRABAJO EN JÓVENES ESTUDIANTES DE ENSEÑANZA MEDIA TÉCNICO PROFESIONAL; COMUNA DE HUALPÉN - CONCEPCIÓN
}

\author{
RODRIGO GANTER SOLÍS ${ }^{1}$
}

MABEL TORNEL CIFUENTES ${ }^{2}$

\begin{abstract}
RESUMEN
El artículo es producto de una investigación tendiente a conocer las expectativas de futuro de jóvenes estudiantes de Enseñanza Media Técnico-Profesional, y los significados otorgados a la educación y al trabajo en dichas proyecciones, considerando como eje conceptual la nueva corriente de estudios de juventud, basada en la articulación de múltiples factores que han renovado y/o transformado la experiencia de ser joven. Los resultados dan cuenta de la estandarización de trayectorias deseadas e imaginadas, donde la historia familiar de precariedad reivindica proyectos de futuro en que la aspiración de una "mejor vida" está directamente vinculada a necesidades económicas, siendo la educación y el trabajo los principales elementos de agencia de que disponen y que movilizarán en función de su cumplimiento.
\end{abstract}

Palabras Clave: Juventud, Trabajo, Educación.

\section{SENTIDOS E SIGNIFICADOS SOBRE A EDUCAÇÃO E O TRABALHO EM JOVENS ESTUDANTES DE ENSINO MÉDIO TÉCNICO-PROFISSIONAL; HUALPÉN - CONCEPCIÓN}

\begin{abstract}
RESUMO
$\mathrm{O}$ artigo é o resultado de uma pesquisa que visa conhecer as expectativas de futuro de jovens estudantes de Ensino Médio Técnico-Profissional e os significados outorgados à educação e ao trabalho nestas projeções, considerando como eixo conceitual a nova corrente de estudos relativos à juventude, baseada na articulação de múltiplos fatores que renovaram e transformaram a experiência de ser jovem.

Os resultados mostram a estandardização de trajetórias desejadas e imaginadas, onde a história familiar de precariedade reivindica projetos de futuro onde a aspiração de uma "melhor vida" está diretamente ligada às necessidades econômicas, sendo a educação e o trabalho os principais elementos de agência que dispõem e que mobilizarão em função de seu cumprimento.

Palavras-Chave: JuVEntude, Trabalho, EducAÇão.

\footnotetext{
${ }^{1}$ Chileno, Sociólogo Universidad de las Artes y las Ciencias Sociales, Doctor en Estudios Urbanos Universidad Católica de Chile, académico Facultad de Ciencias Sociales, Departamento de Sociología en la Universidad de Concepción, correo electrónico: rganter@udec.cl

2 Chilena, Socióloga (Universidad de Concepción), candidata a Magíster en Investigación Social y Desarrollo (Universidad de Concepción), profesional de proyecto Convenio de Desempeño "Sistema Territorial de Educación" en la Universidad del Bío-Bío, correo electrónico: mtornelc60@gmail.com, mtornel@ubiobio.cl
} 


\title{
SENSES AND MEANINGS ON EDUCATION AND WORK IN YOUNG TECHNICAL HIGH SCHOOL STUDENTS; HUALPÉN, CONCEPCIÓN
}

\begin{abstract}
This article is a result of the insight gained from a research on the future expectations of young technical high school students and the meanings they give to education and work in their future plans. The main conceptual axis of this research is the new trend of youth studies, which is based on the articulation of multiple factors renewing and transforming the experience of being young.

The results show a standardization of expectations and future plans in those students whose family histories of scarcity have related, in a direct way, their future aspirations of a "better life" to economic needs, counting on education and work as their main tools to achieve it.
\end{abstract}

\section{KEYWORDS: YOUTH, WORK, EDUCATION.}

\section{INTRODUCCIÓN.}

La conceptualización de la juventud como un proceso de transición lineal, tan característica de las llamadas sociedades "asalariadas", concebía al joven desde una perspectiva eminentemente adulta que privilegiaba su carácter homogéneo y estático, una condición que además "adolecía" de las competencias plenas para insertarse cabalmente y con autonomía en la sociedad. Esto, debido en gran parte a las escasas posibilidades de romper con los ciclos de reproducción social, cuestión que antaño permitía a las familias disponer de ciertas certezas con respecto al futuro de sus hijos. Sin embargo, las transformaciones que comenzaron en el período de posguerra marcaron de manera fundamental la definición y el estudio de la juventud, puesto que las perspectivas que tradicionalmente habían teorizado sobre este fenómeno se volvieron poco a poco obsoletas; nuevos estudios, nuevas corrientes han relevado la condición juvenil como un período influido por múltiples factores que condicionan de manera diversa las experiencias del ser joven.

En este contexto de transformación, que ha dado lugar a una creciente incertidumbre, el estudio de transiciones educativas y laborales de jóvenes estudiantes ha cobrado relevancia debido a la falta de certezas y referentes colectivos que acompañan el vértigo de la sociedad contemporánea, sumado a transformaciones de carácter estructural que han impactado fuertemente en sus proyecciones de futuro y que han hecho emerger una multiplicidad de maneras de "ser joven", muchas veces crecientemente desiguales.

Este artículo detalla los principales resultados de una investigación que tuvo como propósito el estudio de significados que jóvenes estudiantes de Enseñanza Media TécnicoProfesional (EMTP) otorgan a la educación y el trabajo y su vinculación con las expectativas y proyectos de futuro que han configurado en su proceso transicional.

Se concluye la existencia de cierta estandarización de las trayectorias pensadas e imaginadas por los jóvenes entrevistados, en que la educación y el trabajo emergen como vías esenciales para volver palpable un proyecto futuro donde la precariedad que han vivido sus familias y que ha marcado de manera fundamental su biografía y proyección, constituye la base desde la cual movilizan y movilizarán sus recursos disponibles. 


\section{LA EDUCACIÓN MEDIA TÉCNICO PROFESIONAL EN CHILE.}

En nuestro país la situación de la Educación Técnico Profesional es concebida como un pilar fundamental en la formación de capital humano, tanto en el nivel secundario como superior. El Consejo de Retores de CFTs de Universidades pertenecientes al CRUCH señala que esta modalidad tiene como propósito promover transiciones exitosas, tanto hacia el mundo del trabajo como hacia la continuidad de estudios a lo largo de la vida (CRUCH, 2010 en Verdejo, s.f.: 2).

El informe de diagnóstico sobre la Educación Técnico Profesional del MINEDUC (2011) y un estudio denominado "La Enseñanza Media Técnico Profesional en Chile: Orientaciones actuales desde la perspectiva de sus actores", a cargo del Centro de Investigación y Desarrollo de la Educación (CIDE, 2008), concuerdan al describirlo como un sector que agrupa, en su mayoría, a estudiantes de los quintiles inferiores de ingreso y con déficits en indicadores de entrada de logro educacional en relación a aquellos que seguían una educación científico-humanista. Sepúlveda y Ugalde (2010) y Larrañaga et. al. (2014) en estudios realizados con el propósito de describir las trayectorias educativas de estudiantes, así como también sus expectativas, argumentan que el carácter terminal de la educación técnico-profesional ha cambiado para los jóvenes, pues éstos cada vez más esperan continuar estudios superiores una vez terminada su enseñanza media.

Por otro lado, y con respecto a las dificultades que experimenta hoy en día la EMTP, autores como Espinoza et. al. (2011) y Sepúlveda y Ugalde (2010) han dado cuenta de las limitaciones de tiempo y de recursos que evidencian los centros educativos técnicoprofesionales, y de la falta de vinculación entre la formación general y de cada especialidad técnica, además de estar dirigida al ingreso temprano al mundo laboral. Por otra parte, a partir de un informe elaborado por el MINEDUC (2009), se establece que esta modalidad educacional presenta importantes desafíos en términos de calidad, debido a la falta de recursos para el trabajo práctico en los establecimientos $\mathrm{y}$, como dificultad de tipo estructural, se evidencia una brecha entre la EMTP, la oferta técnica en el nivel superior y el mundo productivo, cuestión que desvaloriza el título de nivel medio, a la vez que precariza las posibilidades de inserción laboral y educativa una vez finalizada la enseñanza media.

\section{CONSIDERACIONES METODOLÓGICAS.}

El enfoque metodológico utilizado fue de tipo cualitativo, centrado preferentemente en una modalidad biográfica y dialógica. Se llevaron a cabo 10 entrevistas en profundidad a estudiantes de cuarto año medio del Liceo Pedro del Río Zañartu, comuna de Hualpén, en el Gran Concepción, a través de las cuales se indagó en sus proyecciones de futuro, los factores por los cuales han diseñado dichos proyectos, las estrategias para llevarlos a cabo y los significados que atribuyen a la educación y al trabajo. Para la selección de la muestra se aplicó previamente un cuestionario breve de preguntas abiertas y cerradas a estudiantes de cuarto año de las especialidades de administración y alimentación colectiva dictadas por el establecimiento. Los criterios que se consideraron para la selección fueron:

- Jóvenes que vivieran con ambos padres

- Jóvenes que vivieran con alguno de sus padres 
- Jóvenes en condición de paternidad/maternidad

- Jóvenes que tuvieran alguna experiencia laboral

- Jóvenes sin experiencia laboral

- Jóvenes que hayan señalado querer continuar estudiando en la educación superior

- Jóvenes que hayan señalado no continuar estudios e ingresar al mundo del trabajo

- Jóvenes con ascendencia indígena

La siguiente tabla (Tabla 1) muestra la caracterización de cada uno de los casos, de acuerdo a las variables consideradas en el estudio.

Tabla 1

Caracterización de la Muestra

\begin{tabular}{|c|c|c|c|c|c|c|c|c|c|c|c|}
\hline \multirow{2}{*}{\multicolumn{2}{|c|}{ VARIABLES }} & \multicolumn{10}{|c|}{ CASOS } \\
\hline & & \multirow[t]{2}{*}{1} & \multirow{2}{*}{$\begin{array}{l}2 \\
x\end{array}$} & \multirow[t]{2}{*}{3} & \multirow{2}{*}{\begin{tabular}{|l|}
4 \\
$X$ \\
\end{tabular}} & \multirow{2}{*}{\begin{tabular}{|l|}
5 \\
$x$
\end{tabular}} & \multirow[t]{2}{*}{6} & \multirow[t]{2}{*}{7} & \multirow{2}{*}{$\begin{array}{l}8 \\
x\end{array}$} & \multirow{2}{*}{$\begin{array}{l}9 \\
\mathrm{x} \\
\end{array}$} & \multirow[t]{2}{*}{10} \\
\hline \multirow{2}{*}{ Sexo } & Mujer & & & & & & & & & & \\
\hline & Hombre & $\mathrm{x}$ & & $\mathrm{X}$ & & & $\mathrm{X}$ & $\mathrm{x}$ & & & $\mathrm{x}$ \\
\hline \multirow{3}{*}{ Especialidad } & Al. Colectiva & & & & $\mathrm{x}$ & $\mathrm{x}$ & $\mathrm{x}$ & $\mathrm{x}$ & $\mathrm{x}$ & $\mathrm{x}$ & $\mathrm{x}$ \\
\hline & Administración & $\mathrm{x}$ & $\mathrm{x}$ & $\mathrm{x}$ & & & & & & & \\
\hline & Hotelería & & & & & & & & & & \\
\hline \multirow{4}{*}{ Hogar } & Vive con Padre & & & $\mathrm{x}$ & & & $\mathrm{X}$ & & $\mathrm{x}$ & $\mathrm{x}$ & \\
\hline & $\begin{array}{l}\text { No vive con } \\
\text { Padre }\end{array}$ & $\mathrm{x}$ & $\mathrm{x}$ & & $\mathrm{x}$ & $\mathrm{x}$ & & $\mathrm{x}$ & & & $\mathrm{x}$ \\
\hline & $\begin{array}{l}\text { Vive con } \\
\text { madre }\end{array}$ & $\mathrm{x}$ & & & $\mathrm{x}$ & $\mathrm{x}$ & $\mathrm{x}$ & $\mathrm{x}$ & & $\mathrm{x}$ & \\
\hline & $\begin{array}{l}\text { No vive con } \\
\text { madre }\end{array}$ & & $\mathrm{x}$ & $\mathrm{x}$ & & & & & $\mathrm{x}$ & & $\mathrm{x}$ \\
\hline \multirow{2}{*}{$\begin{array}{c}\text { Pertenencia a pueblo } \\
\text { originario }\end{array}$} & Sí & & & & & & & & & $\mathrm{x}$ & \\
\hline & No & $\mathrm{x}$ & $\mathrm{x}$ & $\mathrm{x}$ & $\mathrm{x}$ & $\mathrm{x}$ & $\mathrm{x}$ & $\mathrm{x}$ & $\mathrm{x}$ & & $\mathrm{x}$ \\
\hline \multirow{2}{*}{ Paternidad/maternidad } & Sí & & & & & $\mathrm{x}$ & & & & & $\mathrm{x}$ \\
\hline & No & $\mathrm{x}$ & $\mathrm{x}$ & $\mathrm{x}$ & $\mathrm{x}$ & & $\mathrm{x}$ & $\mathrm{x}$ & $\mathrm{x}$ & $\mathrm{x}$ & \\
\hline \multirow{6}{*}{ Escolaridad padre } & $\begin{array}{l}\text { Básica } \\
\text { Incompleta }\end{array}$ & & & & & & $\mathrm{x}$ & & & $\mathrm{x}$ & \\
\hline & $\begin{array}{l}\text { Básica } \\
\text { Completa }\end{array}$ & & & & $\mathrm{x}$ & & & & & & \\
\hline & $\begin{array}{l}\text { Media } \\
\text { Incompleta }\end{array}$ & & & $\mathrm{x}$ & & & & & $\mathrm{x}$ & & $\mathrm{x}$ \\
\hline & $\begin{array}{l}\text { Media } \\
\text { completa }\end{array}$ & & $\mathrm{x}$ & & & & & $\mathrm{x}$ & & & \\
\hline & $\begin{array}{l}\text { Superiores } \\
\text { incompletos }\end{array}$ & & & & & & & & & & \\
\hline & No sabe & $\mathrm{x}$ & & & & $\mathrm{x}$ & & & & & \\
\hline \multirow[t]{2}{*}{ Escolaridad madre } & $\begin{array}{l}\text { Básica } \\
\text { Incompleta }\end{array}$ & & & & & & $\mathrm{x}$ & & & $\mathrm{x}$ & \\
\hline & Básica & & & & $\mathrm{x}$ & & & & & & \\
\hline
\end{tabular}




\begin{tabular}{|c|c|c|c|c|c|c|c|c|c|c|c|}
\hline & Completa & & & & & & & & & & \\
\hline & $\begin{array}{l}\text { Media } \\
\text { Incompleta }\end{array}$ & & & $\mathrm{x}$ & & & & $\mathrm{x}$ & & & \\
\hline & $\begin{array}{l}\text { Media } \\
\text { completa }\end{array}$ & & $\mathrm{x}$ & & & $\mathrm{X}$ & & & $\mathrm{x}$ & & $\mathrm{x}$ \\
\hline & $\begin{array}{l}\text { Superiores } \\
\text { incompletos }\end{array}$ & $\mathrm{x}$ & & & & & & & & & \\
\hline & No sabe & & & & & & & & & & \\
\hline \multirow{3}{*}{ Trabajo padre } & Padre trabaja & & & $\mathrm{x}$ & $\mathrm{X}$ & & $\mathrm{x}$ & $\mathrm{x}$ & $\mathrm{x}$ & $\mathrm{x}$ & $\mathrm{x}$ \\
\hline & $\begin{array}{l}\text { Padre no } \\
\text { trabaja }\end{array}$ & & & & & & & & & & \\
\hline & No sabe & $\mathrm{x}$ & $\mathrm{x}$ & & & $\mathrm{X}$ & & & & & \\
\hline \multirow{3}{*}{ Trabajo madre } & Madre trabaja & & & $\mathrm{x}$ & & $\mathrm{x}$ & $\mathrm{x}$ & & & & $\mathrm{x}$ \\
\hline & $\begin{array}{l}\text { Madre no } \\
\text { trabaja }\end{array}$ & $\mathrm{x}$ & $\mathrm{x}$ & & $\mathrm{x}$ & & & $\mathrm{x}$ & & $\mathrm{x}$ & \\
\hline & No sabe & & & & & & & & $\mathrm{x}$ & & \\
\hline \multirow{4}{*}{ Futuro deseable } & Trabajar & & & & & & & & $\mathrm{x}$ & & \\
\hline & $\begin{array}{l}\text { Estudiar en } \\
\text { E.S. }\end{array}$ & & $\mathrm{x}$ & & & & & & & & \\
\hline & $\begin{array}{l}\text { Trabajar y } \\
\text { estudiar }\end{array}$ & $\mathrm{x}$ & & $\mathrm{x}$ & $\mathrm{x}$ & $\mathrm{x}$ & $\mathrm{x}$ & $\mathrm{x}$ & & $\mathrm{x}$ & $\mathrm{x}$ \\
\hline & $\begin{array}{l}\text { Ni trabajar ni } \\
\text { estudiar }\end{array}$ & & & & & & & & & & \\
\hline \multirow{4}{*}{ Futuro posible } & Trabajar & & & & $\mathrm{x}$ & & & & $\mathrm{x}$ & & $\mathrm{x}$ \\
\hline & $\begin{array}{l}\text { Estudiar en } \\
\text { E.S. }\end{array}$ & & $\mathrm{x}$ & & & & & & & & \\
\hline & $\begin{array}{l}\text { Trabajar y } \\
\text { estudiar }\end{array}$ & $\mathrm{x}$ & & $\mathrm{x}$ & & $\mathrm{X}$ & $\mathrm{x}$ & $\mathrm{x}$ & & $\mathrm{x}$ & \\
\hline & $\begin{array}{l}\text { Ni trabajar ni } \\
\text { estudiar }\end{array}$ & & & & & & & & & & \\
\hline
\end{tabular}

Las entrevistas fueron grabadas con el consentimiento de los jóvenes y posteriormente transcritas y analizadas mediante el software Atlas.ti, utilizando como técnica de análisis el método comparativo constante de la Teoría Fundamentada propuesta por Barney Glaser y Anselm Strauss.

\section{MARCO TEÓRICO REFERENCIAL.}

\subsection{Sobre juventud. Visibilización, Conceptualización y Transformaciones.}

Diversos autores han planteado que el concepto de juventud ha sufrido importantes transformaciones, tanto en relación a las características de los jóvenes como del contexto que durante décadas proporcionó marcos de referencia más o menos estables a este grupo generacional. Esto ha repercutido en la teorización y análisis respecto de este segmento de la población, marcado hoy por fuertes tensiones y transformaciones que han repercutido en la manera en que los jóvenes se definen a sí mismos y a sus posibilidades en un contexto 
socio-histórico determinado (Sepúlveda y Ugalde, 2010; Sepúlveda, 2013; Legaspi et. al., 2001; Silva et. al., s.f.), constituyéndose esta nueva realidad en foco de interés para las ciencias sociales, habida cuenta de la obsolescencia de las perspectivas teóricas y metodológicas desarrolladas tradicionalmente y que concibieron a la juventud desde la perspectiva de un proceso de carácter lineal y predecible.

De acuerdo al relato que se ha realizado en relación a la juventud como grupo social, a su visibilización y conceptualización, se señala que ésta es, en primera instancia, una construcción socio-histórica, cultural y relacional de las sociedades contemporáneas, que emerge como una invención de la posguerra (Dávila et. al., 2008). Es sólo en ese momento en que la juventud se visibiliza y comienza a ser definida desde diversas perspectivas y enfoques teóricos.

Desde la conformación de las sociedades modernas industriales, los procesos de conformación de sujetos autónomos se volvieron más complejos. Ello derivó de las necesidades que experimentó el modo de producción capitalista y la organización racional del Estado para su correcta instalación y desenvolvimiento. El tránsito a la vida adulta, en este contexto, estaba marcado por la escuela, el trabajo y la familia, y las diferencias se encontraban asociadas a la posición en la estructura de clases y de sexo, existiendo procesos de reproducción social que se daban en el marco de posibilidades definidas y probables. Esto permitía caracterizar al grupo juvenil de una manera uniforme y cuya transición a la adultez era desarrollada en base a certidumbres, con posibilidades de acción definidas y desenlaces esperables. En este escenario se constituyó la "adolescencia" como el período de tránsito a la vida adulta, conceptualizada como un período de moratoria social, en que se experimentaban cambios a nivel biológico y psicológico, a la vez que comenzaban a ser adquiridos ciertos roles y responsabilidades en la perspectiva de asumir de manera paulatina la "adultez" (Dávila y Ghiardo, 2012). En definitiva, un período vacío y "en espera".

En el período de la postguerra, sin embargo, los límites de la "adolescencia" comenzaron a ser visibles debido a las transformaciones de carácter estructural que tuvieron lugar y que sentaron las bases del nuevo espacio simbólico que permitió el surgimiento de la juventud (Morch, 1996 en Dávila, 2004: 90). La "adolescencia" como construcción histórico-social comenzó a disminuir su capacidad explicativa y comprensiva respecto del fenómeno que comenzaba a darse en la postguerra; grupos de jóvenes comenzaban a experimentar tensiones, dados los crecientes niveles de exclusión producto del desenvolvimiento y desarrollo del capitalismo, constituyéndose este grupo generacional como necesario de control y "adiestramiento". De hecho, la preocupación por la juventud en nuestro país, durante la década del 90, estuvo marcada por el hecho de constituir este grupo social una amenaza para el correcto desenvolvimiento del proceso de transición a la democracia que se estaba instalando, producto de su "apatía". En este escenario, la juventud se volvió "problema" y se transformó en un grupo social necesario de intervención desde el aparato político, mediante el diseño y la aplicación de políticas sociales (Aguilera, 2009).

Esta invención de la postguerra implicó el establecimiento de ciertos límites para el sector juvenil de la población, como por ejemplo de edad, de roles, de responsabilidades, etc. Su emergencia fue acompañada por un creciente interés científico desde diversos enfoques. Desde la sociología, particularmente, el estudio de "juventudes" ha centrado su atención al proceso de integración a la vida adulta (Dávila, et. al., 2008: 53). Como marco estructural, la juventud era percibida como una etapa de la vida en que el paso a la vida adulta se acompañaba de certidumbres respecto de la posición y roles que serían 
desempeñados: la inserción laboral, la conformación de una familia y la independencia pasaban a ser características del adulto, debido a un tránsito más o menos predecible en la etapa de la juventud, a través del cual continuaba el proceso de segmentación social. En este sentido, las familias contemplaban un abanico reducido de posibilidades a las que podrían acceder sus hijos, existiendo mayores probabilidades de heredarles lo que por ellos había sido logrado.

Esta noción de linealidad, de continuidad, de certidumbres respecto del futuro, que caracterizaron la teorización respecto del período de transición juvenil, ha sido cuestionada a través de estudios que han relevado evidencia empírica respecto de nuevos procesos que se han sumado a las experiencias y roles tradicionales del joven, y que han desafiado el aparataje teórico y metodológico tradicional desde el cual se comprendió el proceso de transición a la vida adulta.

Este giro conceptual, que en nuestro país comenzó a gestarse de manera incipiente sólo en la década de los ochenta ${ }^{3}$ (Aguilera, 2009), ha plasmado la discusión en torno a la comprensión de "la juventud" y ha permitido particularizar la experiencia juvenil a través de la constitución de sujetos, de agentes, cuya identidad se construye de manera dialéctica a través de la interacción entre condicionantes estructurales y procesos subjetivos. El resultado: múltiples experiencias, perdiendo la noción de linealidad su tradicional capacidad explicativa. Como argumenta Sepúlveda (2013), quien ha trabajado de manera extensa la temática juvenil en relación a los procesos de transición escuela/trabajo en nuestro país, las conductas, expectativas, proyecciones ya no se encuentran normadas; el paso al trabajo ya no se da de manera lineal, existiendo una serie de procesos en que la subjetividad de los jóvenes da forma a su experiencia juvenil, constituyéndose cada una de ellas en una particular forma de situarse en la sociedad.

La agencia es un elemento que adquiere vital importancia, pues es a través del proceso de interpretación de las circunstancias de la historia personal particular que se elaboran formas específicas e individualizadas de adaptación. Este ejercicio interpretativo, aunque da origen a diversas estrategias que pueden alterar o modificar el curso de vida, recorre límites socialmente estructurados y en el marco de oportunidades y obstáculos que se comportan de manera cambiante (Hitlin y Elder, 2005 en Sepúlveda, 2013: 23).

\subsection{La Educación hoy, ¿Motor de movilidad social?}

De acuerdo a Dávila y Ghiardo (2011: 1192), la escolaridad "es el instrumento de reproducción dominante, el «capital» que más determina la posición que se ocupa en el espacio social y el que más ayuda a reproducirla".

Se ha señalado que los procesos de escolarización obligatoria se han conformado en una de las características centrales de las sociedades contemporáneas, tanto en el nivel institucional como subjetivo. Ello indica que los niveles educativos alcanzados por los jóvenes se constituyen en un elemento central para ellos en tanto grupo social, y en el desarrollo de dicha situación define "una de las características estructurales y subjetivas

\footnotetext{
${ }^{3}$ La juventud en tanto actor social se vuelve objeto de interés y atención de las ciencias sociales en nuestro país sólo a partir de la década de los ochenta, durante la dictadura, existiendo, de acuerdo a Aguilera (2009), dos lecturas teóricas. Por un lado, la juventud como un sujeto parcial, caracterizado por la búsqueda de alternativas, emergiendo con ello una "cultura juvenil", es decir, prácticas específicas y maneras diversas de dar significado a las mismas; por otro lado, la juventud como un sujeto anómico, en que la falta de integración a espacios institucionales formales lleva a acciones más bien individualistas, acompañadas de valores y normas que se van diluyendo.
} 
más relevante de las juventudes chilenas contemporáneas, es decir, su emergencia en la escena social como juventudes escolarizadas” (Molina, 2014: 109).

En nuestro país, y en América Latina de manera generalizada, ha habido importantes avances en términos de cobertura de la educación en sus distintos niveles (Saraví, 2009). De esta manera, sectores antiguamente excluidos de la educación se han visto paulatinamente incluidos a la educación formal. Al respecto, los datos de la CASEN muestran que en 1990 sólo un 60\% de la población de jóvenes de entre 14 y 17 años se encontraba cursando la Educación Media, y en 2013 dicho porcentaje aumentó al 73,3\%. El año 2003, en tanto, marcó un hito fundamental, pues fue en dicho año en que se otorgó obligatoriedad a los doce años de escolarización (Dávila y Ghiardo, 2011). En un estudio llevado a cabo por Dávila et. al. (2005), fueron analizadas las trayectorias sociales y educativas de más de ocho mil estudiantes secundarios y de sus padres en liceos municipales de las comunas de Viña del Mar, Puchuncaví y Quillota. A partir de los resultados obtenidos, los autores concluyen la existencia de cinco tipos de escenarios posibles una vez finalizada la enseñanza media: encontrar un trabajo $(81,6 \%)$; continuar estudios superiores (35,3\%); trabajar y estudiar $(23,7 \%)$; trabajar un tiempo y luego estudiar $(26,6 \%)$; y no lo tengo claro $(14,4 \%)$. Corica (2012), por otro lado, estudió las expectativas educativas y laborales de jóvenes de la escuela secundaria argentina. Los resultados de su investigación señalan que la enseñanza secundaria ya no garantiza, para los jóvenes, una movilidad ascendente o mayores oportunidades de inserción laboral.

Los significativos avances en materia de cobertura escolar, particularmente en el nivel educativo secundario, han permitido a Chile escalar a las tasas más altas de cobertura a nivel latinoamericano. Sin embargo, esta situación no ha sido acompañada de mejoras en los niveles de calidad, ni en términos de aprendizajes ni de la oferta de servicios educativos (Molina, 2004). Es por esta situación que se cuestiona la capacidad movilizadora que se ha asignado a la escuela, "en relación a la importancia estratégica de la educación secundaria tanto para los procesos de desarrollo social, como para la configuración de sus subjetividades y el fortalecimiento de las trayectorias socioeducativas de los sujetos que egresan de la escuela secundaria en Chile y en nuestro continente" (Molina, 2014: 116).

Óscar Dávila y Felipe Ghiardo $(2011 ; 2012)$ han concluido que ha existido en el último tiempo una especie de homogeneización en la estructura de las transiciones de los jóvenes, debido principalmente a las transformaciones que el plano educacional ha experimentado. Así, jóvenes de distintos grupos sociales pasan en la actualidad por las mismas etapas, siguiendo una secuencia más o menos similar, existiendo diferencias en los tiempos que demora cada etapa y las edades a las que se asume cada nueva etapa transicional (Dávila y Ghiardo, s.f.).

Sus hallazgos dan cuenta de las diferencias existentes entre los diversos sectores de la juventud. Éstas tienen que ver con que los jóvenes de los sectores con mayores recursos tienden a continuar estudios superiores de manera inmediatamente seguida al término de la enseñanza secundaria, razón por la cual retrasan los procesos de independencia económica, residencial y familiar. Por otro lado, en los sectores medios y bajos se evidencian trayectos disímiles; algunos continúan estudios superiores, mientras otros ingresan directamente a trabajar y, en este caso, se dan diferencias importantes en términos de sexo en lo que respecta al trabajo remunerado y doméstico. Además, en estos sectores de jóvenes los roles de maternidad/paternidad son asumidos de manera mucho más temprana.

Es en dicho contexto en que los jóvenes experimentan un desajuste entre las expectativas que son cada vez mayores y la realidad de la cual son parte. Los jóvenes de 
menores recursos reconocen las escasas posibilidades de concretar sus aspiraciones, "debido al acceso desigual a las oportunidades de acuerdo con los orígenes sociales familiares, los bajos capitales heredados y la escasa movilidad social que estaría ofreciendo la sociedad chilena" (Dávila y Ghiardo, s.f.: 123). En este sentido, los autores señalan que la escuela como institución formativa no es determinante en la conformación y realización de las expectativas y aspiraciones de los jóvenes, pues éstos se encuentran inmersos en una multiplicidad de instituciones que refuerzan y/o debilitan las proyecciones por ellos realizadas. Así, "todos los antecedentes confirman el rol preponderante de la familia en el desempeño y el logro escolar de los hijos. Los capitales heredados (en general, bajos) o las condiciones de origen de los jóvenes y sus familias marcan diferencias significativas en la configuración de expectativas, aspiraciones y metas educacionales" (Dávila y Ghiardo, s.f.: 124).

\subsection{La Juventud y el Trabajo.}

La crisis económica y social que experimentó el capitalismo liberal y que derivó en la pauperización de las condiciones de vida de una amplia masa de trabajadores fue el hito a partir del cual los estados, ya en la tercera década del siglo XX, asumieron el rol de intervención directa en la economía, tanto en la actividad productiva como en la regulación de las relaciones laborales. Uno de los objetivos fundamentales del Estado-nación era establecer patrones estables y estandarizados que permitieran conducir de manera armoniosa los procesos de desarrollo. La idea de estabilidad otorgada por el proceso de organización se materializaba en la existencia de "referentes claros, coherentes y estables a partir de los cuales construir un sentido de sí mismo, una identidad que debía contribuir a la modernización y que se insertaba (tenía un lugar) en el orden racional y productivo" (Díaz et. al., 2005: 15).

En este contexto, el trabajo ocupó un lugar central producto del establecimiento de una nueva relación laboral, la relación salarial, que otorgó al trabajo un carácter duradero, constituyéndose éste en la "base para el reconocimiento social y de las protecciones contra la inseguridad y la desgracia" (Castel, 1997: 403), y fue el principal mecanismo bajo el cual los individuos desarrollaban un sentimiento de pertenencia social por su inserción en una colectividad hacia la cual era leal, permitiendo esto "compartir un núcleo de símbolos y representaciones sociales y, por lo mismo, una orientación común a la acción” (Giménez, s.f.: 11).

Ha existido en el último tiempo un creciente interés por estudiar la manera en que los procesos de transformación estructural han repercutido en la configuración de las trayectorias juveniles. La ruptura con las antiguas experiencias juveniles, hoy ha permitido la emergencia de nuevos y más difíciles procesos de inserción social, a partir de los cuales se ha argumentado la existencia de una transformación que ha afectado a todos los ámbitos sociales y a quienes circunscriben su vivencia en ellos. En este sentido, el mundo del trabajo ha experimentado un giro trascendental, en línea con la instauración de nuevos mecanismos de organización social, económica, política, cultural, que más que promover el desarrollo de todo y todos, sólo han transformado las tradicionales formas de exclusión, a través de una "sofisticación" de los filtros de aceptación.

La flexibilidad, la segmentación, la precariedad con que hoy se vive la experiencia laboral ha provocado un desplazamiento del trabajo, su descentramiento, situación que ha sido acompañada de mecanismos que obligan a la escolarización extendida, muchas veces 
visualizada como un obstáculo a los horizontes de bienestar ansiados por los jóvenes y los grupos sociales en general, dadas las dificultades que representa la obtención de un título técnico o profesional.

Hoy, también los jóvenes están transformando su relación con el mundo del trabajo, emergiendo con esto una diversidad de trayectorias, muchas de las cuales están permeadas por los signos de la exclusión y por lógicas de acción individuales.

Weller (2006), en el marco de un análisis comparativo de distintos estudios referidos al sentido del trabajo en países de América Latina, señala que los jóvenes perciben hoy grandes dificultades para insertarse laboralmente. Además, el encontrar un trabajo no significa inserción plena, pues las primeras experiencias laborales se caracterizan por altos grados de precariedad y discontinuidad. Este análisis está también presente en una investigación llevada a cabo en las comunas de Santiago, Valparaíso y Concepción. Sus resultados dan cuenta de zigzagueantes experiencias laborales, de cambios de rama de actividad, importantes reconversiones ocupacionales, situaciones a partir de las cuales los jóvenes ven eternizado su proceso de conformación como sujetos autónomos. Los primeros contactos con el mundo laboral, que para los jóvenes comienzan siendo "pegas chicas", se configuran poco a poco en una condición permanente, que impide a los jóvenes trazar proyectos de vida en el mediano y largo plazo (Ghiardo, 2009).

Con respecto a los significados que son otorgados al trabajo, Weller (2006) señala que los jóvenes con altos niveles socioeducativos tienden a la búsqueda de trabajos que sean de su gusto, que les brinden remuneraciones atractivas y que les otorguen reconocimiento personal. Por otro lado, en los grupos con bajos niveles socioeducativos, el trabajo es significado como un espacio que debiera operar sobre la base del respeto y la dignidad, siendo el esfuerzo una característica central. En la misma dirección, Sepúlveda (2004), en un estudio realizado con jóvenes de las comunas de Melipilla y San Ramón en la región Metropolitana, concluyó que, a pesar de existir condiciones estructurales que limitan las posibilidades de agencia de los sujetos, a partir de la construcción y realización efectiva de proyectos de futuro, el esfuerzo personal es caracterizado como un mecanismo importante para la superación de dichas dificultades.

Frente a ese escenario, los jóvenes deben ingeniar estrategias individuales, echando mano a los recursos de que dispongan, siendo el capital social un recurso clave a través del cual los jóvenes visualizan posibilidades de integración a un puesto de trabajo. Sin embargo, se señala su carácter ilegítimo y limitado, debido a que atenta contra el principio meritocrático, por un lado, y en algunos casos las redes son recursivas y no ascienden, dificultando esto la progresión de los jóvenes en el ámbito laboral (Ghiardo, 2009).

Si bien ha ocurrido un descentramiento del trabajo, sobre todo en las generaciones jóvenes, éste aún constituye un eje sobre el cual es posible enrolar proyectos de vida, así como también un posicionamiento que es clave en la realización del bienestar, personal y material.

\section{RESULTADOS Y ANÁLISIS.}

\subsection{La Educación, el Trabajo y la Familia... más la ilusión de un "Vivir Mejor".}

Los y las jóvenes, especialmente quienes han sufrido la ausencia de alguno de sus padres, caracterizan a quienes los han criado y formado en el ámbito familiar como el principal ejemplo a partir del cual han conformado sus proyectos y expectativas de futuro. En éstos, 
la educación es señalada como el vehículo fundamental a través del cual les es posible romper el círculo familiar, ligado al "no tener lo suficiente", por ejemplo. Los padres de todos los y las jóvenes entrevistadas no accedieron a estudios superiores, algunos completaron la enseñanza básica y otros sólo llegaron a algunos niveles de la educación media, razón por la cual es añorado el optar por, y lograr tener, un título profesional. Esto, para los padres y los y las jóvenes, es causa de las necesidades que han debido pasar, debido al tipo de trabajo al que es posible optar con escasos niveles de cualificación. Por otra parte, la educación superior es símbolo de una ruptura con la historia familiar, caracterizada por precariedades y dificultades aún más acentuadas.

El relato respecto de aquellas cosas que son señaladas por los y las jóvenes como proyectos y expectativas de futuro se encuentra fuertemente asociado a las ideas de trabajo, familia y tener una casa propia. Esta trayectoria imaginada y deseada se encuentra influida, y a la vez tensionada, por el ejemplo de sus familias, y de sus padres en particular, cuyas carencias e imposibilidades han moldeado en estos jóvenes el deseo de superar lo que sus padres pudieron lograr. En este sentido, no es sólo la propia biografía la que incita la imaginación y evoca expectativas, sino también, y de manera fundamental, su historia familiar. En realidades de precariedad y exclusión, por tanto, "vivir mejor" es un anhelo, pero también una obligación. "Querer ser alguien en la vida" es, en definitiva, la materialización de las expectativas de padres y madres que ven en sus hijos e hijas la posibilidad de romper con el ciclo del que han sido parte.

Aquellos jóvenes que son padres/madres dan cuenta de proyectos de futuro necesarios de llevar a cabo en el corto plazo, debido a la urgencia que representa el tener hijos. Este escenario incentiva en los jóvenes la generación de estrategias de corto plazo que pueden llevar al abandono de aquellos proyectos y expectativas pensadas antes de asumir estos nuevos roles.

En relación al cómo hacer concreto dicho proyecto, la idea de trabajo es erigida por los y las jóvenes a la vez como un medio y como un fin. En este sentido, si bien el trabajo ha experimentado una serie de transformaciones, continúa siendo un mecanismo central en los procesos de inclusión social de la juventud. Sin embargo, el sentido que adquiere hoy en día es incuestionablemente diferente, y esto se refleja en que el trabajo es, por sobre todo, algo necesario de realizar en función de obtener ingresos que permitan "vivir mejor", "tener una mejor calidad de vida", "sustentarse". Ligado a esto, la educación es antecedente fundamental en el proceso de constitución juvenil; una promesa por la que se apuesta mayoritariamente, una "puerta" que es necesario abrir y que amplía el espectro de posibilidades a las que les es posible optar.

Por otra parte, al analizar los proyectos y mecanismos que son visualizados por los y las jóvenes como las vías por las que es posible transitar para concretar sus aspiraciones, lo que finalmente se vislumbra es una presión excesiva sobre jóvenes que son los que en mayor medida requieren de soportes y apoyo concretos. La individualización es, para estos jóvenes, clave para hacer sostenibles y materiales sus proyectos, cuestión que es reflejada en la responsabilidad auto asumida de ser ellos y ellas quienes gestionen recursos y los movilicen en función de sus propios propósitos. No hay reclamos, sino la asunción de ser aquélla "la" vía para, finalmente, hacer sus vidas.

\subsection{Sustento y vocación: Expectativas sobre el Trabajo.}


El trabajo es visualizado por los y las jóvenes como un medio para alcanzar objetivos que exceden las fronteras de la actividad laboral, en tanto actividad fundamental a partir de la cual les es posible construir sus trayectorias imaginadas y así superar los límites que sus padres y cercanos han experimentado. "Si uno no trabaja en esta vida no es nada" señala una de las jóvenes entrevistadas, refiriéndose con ello a que el trabajo es la vía obligada a través de la cual es posible lograr la independencia. Es también reflejo de un logro, de recompensas obtenidas a partir del esfuerzo que individualmente han impreso en el proceso de consecución y cumplimiento de sus metas. Como jóvenes, señalan que el trabajo es un medio para comenzar a "hacer una vida" y hacer de la misma algo sostenible por la vía de utilizar y movilizar los recursos de que dispongan y generar de manera individual estrategias para escalar. Ante las urgencias económicas que han caracterizado la vivencia de su biografía, la obtención de ingresos se vuelve premisa en la gestación de sus proyectos.

Al proyectar su futuro, los y las jóvenes ponen énfasis en el desarrollo de actividades laborales relacionadas a aquello que les gusta, que les permita lograr cierta realización personal. Si bien es importante conseguir un trabajo bien remunerado, para ellos es también fundamental dar continuidad a sus actividades laborales en función de lo que les apasiona hacer. Por ello, el sentido que otorgan al trabajo está mediatizado por las oportunidades educativas a las que puedan acceder, buscando con ello el reconocimiento formal, la acreditación de su vocación, y a partir de aquello ampliar el abanico de posibilidades laborales.

\subsection{Romper el círculo familiar: Eje de expectativas educativas.}

Los y las jóvenes constantemente se refieren a los estudios superiores como una especie de puerta que abre oportunidades, "el primer paso para abrir todas las puertas que siguen", dice una joven entrevistada, sin embargo, enfatizan en lo dificultoso que es dedicar tantos años a prepararse en algo que posiblemente no dé los frutos esperados de manera inmediata. Señalan los jóvenes que el hecho de trabajar en lo que guste, después de tanto tiempo de estudiar, es una cuestión difícil. Esto da luces respecto de la existencia de cierta crítica desde los y las jóvenes, quienes reconocen la necesidad, sino la obligación, de estudiar y certificar sus conocimientos por la vía de un proceso de escolarización formal excesiva.

A pesar de aludir de manera constante a cierto juego estratégico para ingresar y mantenerse en el mercado laboral, por medio de la adaptación, del buen comportamiento, del sentido de responsabilidad, etc., existe entre los y las jóvenes el reconocimiento respecto de la discriminación de la que son parte aquellos jóvenes que, voluntariamente o no, no han accedido a estudios superiores. De esta manera, la educación técnico profesional, si bien es considerada un "respaldo" ante la imposibilidad de continuar estudios, los jóvenes se reconocen en desventaja debido a las posibilidades de empleo que un título de técnico de nivel medio les brinda.

La situación de incertidumbre se agudiza en los jóvenes cuando han vivenciado de manera indirecta la frustración de familiares que, aun cursando estudios superiores, no han podido desarrollarse de manera plena en aquello para lo cual estudiaron, emergiendo en los y las jóvenes proyectos quizás menos ambiciosos, sobre todo cuando existe la obligación de mantener una familia. 
Sin embargo, la educación es la herramienta más preciada para conseguir una mejor calidad de vida, y es el vehículo que sus padres han señalado como el correcto. Relatan que sus padres añoran que consigan estudiar, que esto es lo único que ellos les pueden dejar.

Hoy en día, la inserción en trabajos de calidad requiere del reconocimiento formal de conocimientos y habilidades, a través de la obtención de títulos otorgados por la educación superior, cuestión que se ha instaurado como premisa en la juventud, sobre todo en aquellos sectores provenientes de contextos en que reina la precariedad y la urgencia económica. "Ser alguien en la vida" es el mega proyecto de todos los y las entrevistadas, y es la educación el recurso privilegiado para concretarlo. A través de ella es que conciben posible desarrollarse laboralmente y, por fin, conseguir ingresos suficientes para vivir, y vivir bien.

Señalan que la opción de estudiar debe ser acompañada de trabajos complementarios, debido al deseo de ayudar a sus familias y a quienes costearán sus estudios superiores para no ser una "carga" y así alivianar el importante gasto que estudiar significa, sobre todo considerando sus condiciones socioeconómicas. Aun teniendo posibilidades de dedicar su tiempo sólo a estudiar, como se lo han propuesto familiares con el propósito de que puedan focalizar sus esfuerzos en un objetivo concreto, señalan que es importante actuar de manera independiente, cuestión que realizarían por medio de actividades laborales complementarias, respondiendo en gran medida al ejemplo que sus padres y madres les han brindado, cobrando esto un sentido particularmente especial en aquellos jóvenes hijos de padres/madres solteros. Por otro lado, se encuentra plasmada en los discursos de los y las entrevistadas la idea de la independencia, materializada en la satisfacción propia de necesidades vinculadas a la compra de materiales que puedan ser demandados en el transcurso de sus estudios, así como también a actividades que desarrollen en su cotidianeidad sin depender del resto.

\subsection{El respaldo que brinda la Educación Media Técnico Profesional.}

Los y las jóvenes reconocen diferencias entre las modalidades de enseñanza media científico-humanista y técnico-profesional, sin embargo enfatizan un cierto criterio estratégico al optar por la educación técnica, al obtener con ésta "un pequeño título", un "piso" o "algo" con qué enfrentar la vida si no se pudiese a optar a estudios superiores o "si llegara ocurrir algo" que les impidiera seguir estudiando, puesto que en el área científicohumanista se "juega todo a una sola cosa", que es el ingreso a la educación superior. En el transcurso de aquel proceso, podrían "aburrirse" o "hacer otra cosa", quedando a la deriva, sin nada que pudiera otorgarles algún suporte más concreto. En la enseñanza técnica, si bien no son preparados de manera integral para rendir la PSU, obtienen conocimientos ligados a un ámbito que, en la mayoría de los casos de jóvenes entrevistados, tiene que ver con lo que efectivamente quieren hacer en un futuro.

Si bien el título de técnico de nivel medio no les asegura en el corto plazo oportunidades bien definidas para optar a un trabajo, valoran el hecho de contar con conocimientos en ámbitos prácticos de sus áreas de interés (especialidades). Por otro lado, confían en la experiencia que significa realizar su práctica profesional, lo que caracterizan como una oportunidad de permanecer en el lugar en que la desarrollan, cumpliendo con responsabilidad lo que les es demandado.

Reconocen que el título de técnico de nivel medio es un "pequeño título", que no asegura posibilidades como sí lo hace en mayor medida un título profesional. Por ello es 
que asumen que sus primeras experiencias laborales serán más bien precarias y que deberán realizar actividades distintas de las que estudiaron, y que por ello deberán "escalar de a poco", mostrando un buen comportamiento, siendo responsables, puntuales, etc.

$\mathrm{Si}$ bien se reconocen diferencias entre un título de nivel medio y uno superior, otorgan especial confianza a su capacidad de gestionar los recursos con que cuentan, relacionados fundamentalmente a sus atributos personales (actitudes, valores, conocimientos), para con ello lograr cierto reconocimiento y a partir de aquello optar a posibilidades de empleo más cercanas a lo que esperan laboralmente.

\subsection{Sentidos en torno al Trabajo.}

Los y las jóvenes concuerdan al describir un escenario laboral mayormente dificultoso, en que encontrar un trabajo que cumpla con sus expectativas se encuentra condicionado por una serie de factores que exceden los recursos con los que individualmente cuentan. En este sentido, mencionan la "competencia" existente hoy en día por una gran cantidad de profesionales en búsqueda de trabajo, con similares títulos, en un mercado incapaz de absorber tal nivel de profesionalización.

Importante es la concepción que tienen respecto de las posibilidades que brinda el estar "apitutado". Éste es caracterizado por los jóvenes como un recurso fundamental y primario para el proceso de inserción laboral, sin embargo, a pesar de lo estratégico que resulta el disponer de contactos, concuerdan en señalar que recurrir al "pituto" resulta ser más bien incorrecto, pues debieran ser las capacidades con que cuentan y no las redes las que primen al momento de seleccionar a quiénes ocuparán un puesto de trabajo.

Por otro lado, emerge de los relatos un dejo de desilusión ante un mercado laboral que privilegia ciertas áreas de especialización, en desmedro de otras, dando cuenta también de una crítica a la jerarquización de los niveles educativos, reflejada en las distancias salariales existentes entre un título universitario y aquellos obtenidos en la educación técnica secundaria y superior. Estas situaciones, al decir de los y las jóvenes, obstaculizan el logro de su independencia y, más aún, la posibilidad de comenzar a hacer una vida.

Caracterizan el proceso de inserción como un quiebre, una experiencia completamente diferente a las que han tenido a lo largo de sus vidas, en que los errores ya no serán corregidos, sino más bien sancionados y "costarán caro". La "adaptación" es, en este sentido, clave. En torno a ello, señalan que mantener un puesto de trabajo demanda de los y las jóvenes un aprendizaje vinculado a formas de comportamiento definidas, en que es primordial ser estratégico, sobre todo en lo que refiere a relaciones laborales, en que el "chaqueteo" emerge como una característica propia de los ambientes de trabajo.

Señalan con recurrencia que son las características individuales, y particularmente sus comportamientos y actitudes, las "competencias blandas", los factores que predominarán en su ingreso y estabilidad en el trabajo. El caso de la joven con ascendencia indígena da cuenta de limitantes que exceden su propio control. En este sentido, señala que en muchas ocasiones ella ha visto y vivido cómo las cosas se vuelven más engorrosas cuando se es indígena, lo que asegura será un factor que también limitará sus posibilidades a la hora de ingresar al mundo laboral. Si bien de su discurso se desprende la existencia de más presiones y obstaculizadores ligados a la discriminación con que jóvenes indígenas deben lidiar, reivindica la capacidad que ha generado y a la que ha de recurrir para "mostrarse capaz". 
Entre los jóvenes entrevistados no existe apelación a entidades u organismos que daban asumir alguna responsabilidad en el proceso de inserción laboral. El problema radica, más bien, en la competencia existente por puestos de trabajo, recayendo sobre los propios jóvenes la responsabilidad de "competir" de buena manera, por medio de un "buen comportamiento" y un adecuado proceso de adaptación. En este sentido, los y las jóvenes reivindican la importancia que tiene el "esfuerzo", tanto para ingresar a un puesto de trabajo, como para ascender una vez insertos. "Cuidar el trabajo" es reflejo de esta idea del esfuerzo, en que la competencia a la cual refieren debe ser resuelta individualmente.

Además de las actitudes y de los comportamientos que mencionan como relevantes a la hora de encontrar un trabajo, señalan también que la experiencia, así como también el reconocimiento son factores que, suponen, predominan en la decisión que pudieran tomar quienes se encuentran a cargo de completar puestos de trabajo. Ésta es una cuestión fundamental, puesto que desde este argumento es que los y las jóvenes caracterizan un escenario muy complejo. En tanto no se les dé la oportunidad para insertarse al mundo del trabajo, ¿de qué manera cumplir con los criterios de experiencia?, ¿de qué manera lograr reconocimiento? Éstas son preguntas que constantemente plantean de manera indirecta todos los entrevistados, y la reflexión que realizan al plantearse dichas preguntas es la concepción del "riesgo". En este sentido, señalan que a empresarios o personas a cargo de reclutar a trabajadores les resulta arriesgado contratar a alguien sin experiencia, debido a los errores en que podrían incurrir. Como respuesta a esta situación, nuevamente la "educación avanzada" constituye una salida, debido a los conocimientos con los que debiera contar cualquier persona que ha estudiado prolongadamente.

La experiencia es una cuestión que relevan de manera constante $\mathrm{y}$, por tanto, deben "empezar de a poco", asumiendo con ello que podrían comenzar en labores muy por debajo de sus expectativas. "Empezar de a poco" es descrito como una especie de recurso al que deben recurrir, pues sólo a través de ello podrán demostrar aquellas capacidades, comportamientos, valores con que, de acuerdo a sus relatos, podrán posteriormente "ascender".

\section{CONCLUSIONES}

A partir de los cambios que ha experimentado la experiencia juvenil, la desestandarización, la individualización y la incertidumbre han emergido como características del colectivo joven. Sin embargo, de acuerdo a los resultados obtenidos en esta investigación, los y las jóvenes destacan ámbitos significativos de sus proyectos de futuro en función de ideas y concepciones propias de otra época, aunque con tintes e implicancias claramente diferenciados. La familia, la educación y el trabajo son nombrados como pilares de un proyecto futuro alimentado fundamentalmente por la ilusión de una vida mejor, en la perspectiva de un proceso lineal en que de estudiar se espera pasar a trabajar para construir una base económica que permita la conformación de una vida independiente y en familia. Son estos los hitos que conciben no sólo como experiencias a las que deberán optar, sino también como recursos que esperan movilizar en función de las expectativas de futuro que han elaborado.

El sentido otorgado tanto a la educación como al trabajo radica en las posibilidades que estas actividades o ámbitos experienciales brindan en función de la superación de una historia familiar de precariedad, basada en la carencia económica. De acuerdo a lo planteado por Dávila et. al. (2008), Hitlin y Elder (2005 en Sepúlveda, 2013) y Casal et. al. 
(2011), entre otros, el tiempo presente de los jóvenes no está determinado sólo por la acumulación de experiencias pasadas, sino también por las aspiraciones de futuro, las que son interpretadas de manera individual, dando lugar a un proceso de elaboración de estrategias y recursos que permitan convertir un escenario futuro en algo posible de palpar. Sin embargo, este proceso de creación, de "imaginación", se ve obstaculizado por una trayectoria que obliga a pensar desde "lo que no se tuvo".

La carencia es, entre estos jóvenes, la base desde la cual han elaborado un escenario futuro y estrategias para hacerlo palpable. "Tener una mejor vida que la que tuvieron mis padres", "lograr ser mejor que ellos", "tener más cosas" son expectativas que, si bien reflejan un "querer ser", se encuentran asociadas más a la negación o más bien superación de una historia familiar de precariedades.

Se desprende de la proyección que realizan las limitaciones con que es imaginada una trayectoria, en que poco es el espacio que queda para "imaginar" o exceder un futuro en que lo económico se vuelve más una obligación.

¿Dónde se encuentra la agencia? Las problemáticas económicas que han afectado la biografía de los y las jóvenes los ha llevado a generar proyectos cuya base es la obtención de mayores ingresos, estableciendo a la educación y el trabajo como el recorrido a través del cual es posible lograr una vida mejor. Este escenario podría ser la base de un argumento que niegue la agencia de estos jóvenes. Sin embargo, los resultados de esta investigación nada tienen que ver con eso. A pesar de "verse obligados" y estar fuertemente determinados $\mathrm{y}$ anclados por la posición que ocupan en la "cancha social", y a ser parte del juego asfixiante de la escolarización, estos jóvenes han tomado opciones biográficas valiosas a través de su trayectoria y con ello en el propio diseño de un horizonte futuro posible, aunque indeterminado, donde no se ven renunciando en sus empeños, sino peleando, orientados en hacer lo correcto y no desviarse de su meta, maniobrando en la adversidad y en medio de contextos barriales donde muchas veces resulta más lucrativo la obtención de ingresos y prestigio por los caminos que contravienen lo legal. La educación y el trabajo, como fue señalado, no son sólo proyectos de corto o mediano plazo, sino los recursos de que disponen para movilizarse y escalar social y económicamente.

En este sentido, los y las jóvenes han decidido; han optado por emprender trabajos de acompañamiento mientras estudian, han concebido la obtención de un título de técnico de nivel medio como "soporte" en caso de no acceder a estudios superiores. Esto no es sólo un juego adaptativo a la obligación que el sistema genera, sino la gestión activa y relacional de recursos, capitales y competencias que han hecho propios y visualizado como tendientes a la consecución de sus propósitos.

La educación, por un lado, es condicionante estructural, a la vez que subjetivo (Molina, 2014). "La educación abre puertas" es una concepción que los jóvenes asumen como verdad, pero esto emerge no como "lo que estoy obligado a hacer", sino como el recorrido por el que deben realizar su transición y así hacer concreta la trayectoria imaginada.

Por otro lado, el trabajo es considerado "la vía" a través de la cual es posible vivir y que, ya finalizada su enseñanza media, han comenzado a visualizar desde una perspectiva estratégica, en que sólo depende de ellos y ellas ingresar y escalar laboralmente. La adaptación, el buen comportamiento, el esfuerzo, el compromiso, el entregarse, es transversal en los relatos de todos los entrevistados, y éstos son pensados en clave de agencia, de apropiación. 
Si bien es la precariedad la que lleva a estos jóvenes a imaginar su futuro, de ningún modo esto los inhabilita. Contrario a ello, y a pesar de ser las agencias tradicionales (la educación y el trabajo) los recursos a los cuales aluden para volver material la idea de una mejor vida, los y las jóvenes ciertamente han hecho propia una historia familiar de precariedad, que los ha incentivado a actuar conforme a la superación, el esfuerzo colectivo de las familias y el logro personal.

A pesar de todo lo anterior, observamos en el escenario más estructural y global que contiene las experiencias y biografías descritas hasta aquí, el predominio actual de un contexto social de profundos desafueros y marcadas expulsiones del sistema, donde se hace muy difícil sostener las promesas y garantías que en otro momento social otorgó el soporte de la escuela y el trabajo a muchos de estos jóvenes. En medio de la fuerte retirada de las coberturas que en algún otro momento histórico otorgó la llamada matriz nacional-popular en Chile, observamos la actual desprotección de muchos de estos jóvenes y por tanto los desiguales modos de vivir lo juvenil en el Chile de la era neoliberal, donde sólo para algunos se vivencia la condición "privilegiada" de moratoria social, pues otros se ven forzados a dejar dicha condición para asumir tempranamente las tareas de la paternidad y la maternidad, o bien, el ingreso temprano a mercados laborales altamente precarizados. Y lo hacen mientras estudian, lo hacen en medio de mínimas coberturas institucionales, y lo asumen como desafíos exclusivamente personales, aumentando la presión social sobre ellos mismos, y desplazando las responsabilidades sociales e institucionales del éxito o del fracaso en sus trayectorias de movilidad, también sobre sí mismos. Lo que no significa que estos jóvenes no visualicen un futuro, un conjunto de expectativas y sueños, sino que en su diseño dicho horizonte aparece eclipsado por factores de origen que incrementan la sensación de incertidumbre, no así la confianza en ellos mismos. Además de una potente pulsión por ser mejor que sus padres y no defraudarlos.

Por todo lo anterior, en un cierto nivel del análisis, coincidimos con quienes plantean que asistimos actualmente a un proceso de des-juvenilización (Valenzuela, 2009), sobre todo en los componentes sociales y en los actores que observamos en este estudio, pues reconociendo que la dimensión de agencia no se pierde en ellos, sostenemos preliminarmente una tensión no resuelta entre las competencias que estos jóvenes y sus redes generan para poder navegar en la adversidad, y las fuertes corrientes estructurales que los van expropiando de la esperanza y las oportunidades ${ }^{4}$. Esta tensión, que incluye dos tendencias que reflejan significativos hallazgos de este estudio, nos parece central para poder problematizar e integrar de modo más sistemático en futuros estudios en torno a la condición de lo juvenil abordada en esta propuesta. Sobre todo frente a las prioridades y los desafíos que debe encarar en los años siguientes la formación técnico - profesional de los jóvenes en Chile; y los sentidos que éstos construyen en torno a la educación y el trabajo como recursos y herramientas que deben volver a recuperar el peso que en algún otro momento tuvieron, en tanto dispositivos genuinos y sostenibles de movilidad social.

\footnotetext{
${ }^{4}$ Aquí asumimos los procesos de des-juvenilización, no como procesos mediante los cuales se les expropia por completo la condición juvenil a los sujetos, sino más bien como procesos estructurales mediante los cuales muchos jóvenes se ven expuestos -a pesar de las dinámicas de agenciamiento- a mecanismos de desafuero de garantías y oportunidades para constituirse plenamente como sujetos autónomos. Dichos procesos se hacen más visibles en el contexto de un particular modo de producir y gestionar las juventudes, con una fuerte y radical orientación neoliberal, como el caso chileno, que refuerza la exclusión y la desigualdad social, por tanto las múltiples -y muchas veces veladas- formas del desafuero y la precarización biográfica.
} 
CONCEPCIÓN (CHILE), ENERO 2016

RECIBIDO: ENERO, 2016

ACEPTADO: JULIO, 2016

\section{REFERENCIAS BIBLIOGRÁFICAS}

AGUILERA, O. (2009). "Los estudios sobre juventud en Chile: coordenadas para un estado del arte". Revista Última Década $\mathrm{N}^{\circ} 31$. Valparaíso: CIDPA.

CASAL, J.; MERINO, R. \& GARCÍA, M. (2011). "Pasado y futuro del estudio sobre la transición de los jóvenes". Papers Revista de Sociología No 96. Disponible en: http://papers.uab.cat/article/view/v96-n4-casal

CIDE (2008). "La Enseñanza Media Técnico Profesional en Chile: Orientaciones actuales desde la perspectiva de sus actores". Disponible en: http://biblioteca.uahurtado.cl/ujah/reduc/pdf/pdf/txt828.pdf 20 de Abril de 2014]

CORICA, A. (2012). "Las expectativas sobre el futuro educativo y laboral de jóvenes de la escuela secundaria: entre lo posible y lo deseable”. Última Década No 36 . Valparaíso: CIDPA.

DÁVILA, O. (2004). "Adolescencia y juventud: de las nociones a los abordajes". Última Década $\mathrm{N}^{\mathrm{0}} 21$. Valparaíso: CIDPA.

---- \& Ghiardo, F. (2005). "Trayectorias, transiciones y condiciones juveniles en Chile". Nueva Sociedad $\mathrm{N}^{\circ}$ 200. Disponible en: http://nuso.org/articulo/trayectoriastransiciones-y-condiciones-juveniles-en-chile/

(2011). "Trayectorias sociales juveniles. Cursos y discursos sobre la integración laboral”. Papers Revista de Sociología Vol. 96 №4. Disponible en: http://papers.uab.cat/article/view/v96-n4-davila/papers 96_4-davila

------- ------- (2012). "Transiciones a la vida adulta: generaciones y cambio social en Chile". Revista Última Década No 37. Valparaíso: CIDPA.

--------- \& Medrano, C. (2008). Los desheredados. Trayectorias de vida y nuevas condiciones juveniles. Valparaíso: Ediciones CIDPA (cuarta edición).

DÍAZ, X.; GODOY, L. \& STECHER, A. (2005). "Significados del trabajo, identidad y ciudadanía. La experiencia de hombres y mujeres en un mercado laboral flexible". Santiago: Centro de Estudios de la Mujer. Recuperado en http://www.cem.cl/pdf/cuaderno3.pdf

ESPINOZA, O., CATILLO, D.; TRASLAVIÑA, P. (2011). La implementación de la Reforma Curricular en la Educación Media Técnico Profesional: Evaluación y Proyecciones. Santiago: CIE, PIIE, Universidad UCINF.

GIMÉNEZ, G. (s.f.). Materiales para una teoría de las identidades sociales. México: Instituto de Investigaciones Sociales de la UNAM. Recuperado en http://docentes2.uacj.mx/museodigital/cursos_2008/maru/teoria_identidad_gimenez.p df [10 de agosto de 2011]

GHIARDO, F. (2009). "Transformaciones del trabajo y juventud: temas y búsqueda". Última Década, № 31. Valparaíso: CIDPA.

LARRAÑAGA, O.; CABEZAS, G. \& DUSSAILLANT, F. (2014). "Trayectorias educacionales e inserción laboral en la enseñanza media técnico profesional". Estudios públicos $\mathrm{N}^{\mathrm{o}}$ 134. Disponible en: http://www.cepchile.cl/trayectorias- 
educacionales-e-insercion-laboral-en-la-ensenanza-media/cep/2016-0304/100655.html

LEGASPI, L., MONEDERO, F., BATLLE, S. \& AIESENSON, D. (2001). "Trayectorias educativas y laborales de jóvenes en transición de la escuela al estudio y/o el trabajo". $5^{\circ}$ Congreso Nacional de Estudios del Trabajo. Buenos Aires. Disponible en: http://www.aset.org.ar/congresos/5/aset/PDF/AISENSONYOTROS.PDF

MINEDUC (2009). Bases para una política de formación técnico profesional en Chile. Comisión para el estudio de la formación técnico profesional en Chile, Informe Ejecutivo. Santiago.

- (2011). "Educación técnica profesional en Chile. Antecedentes y claves de diagnóstico". Disponible en:

http://www.mineduc.cl/usuarios/mineduc/doc/201204241130130.DiagnOsticoEducac iOnTPCentrodeEstudiosMINEDUC.pdf [20 de Abril de 2014]

MOLINA, W. (2014). "Juventudes y procesos de escolarización secundaria en el Chile contemporáneo". En Pablo Cottet, Juventudes. Metáforas del Chile contemporáneo ( $1^{a}$ Ed.). Santiago, Chile: Ril Editores.

SARAVÍ, G. (2009). "Juventud y sentidos de pertenencia en América Latina: causas y riesgos de la fragmentación social". Revista CEPAL No 98. Disponible en: http://repositorio.cepal.org/bitstream/handle/11362/11295/098045065 es.pdf?sequen ce $=1$

SEPÚLVEDA, L. (2004). "Expectativas y estrategias laborales de jóvenes y adultos jóvenes en Chile". En de Groote M. y Weller, J. (Eds). Juventud y mercado laboral: brechas y barreras. Santiago: FLACSO-CEPAL.

SEPÚlVEDA, L. (2013). "Juventud como Transición: Elementos Conceptuales y Perspectivas de Investigación en el Tiempo Actual". Última década $\mathrm{N}^{\circ} 21(39)$. Valparaíso: CIDPA.

SEPÚLVEDA, L. \& UGALDE, P. (2010). "Trayectorias disímiles y proyectos individualizados. Origen y experiencia educativo-laboral de los estudiantes de centros de formación técnica". Calidad en la educación $\mathrm{N}^{\circ}$ 33. Santiago: Consejo Superior de Educación.

SILVA, I., CONTRERAS, C. MARTÍNEZ, T. \& SEPÚLVEDA, M. (s.f.). "Expectativas de futuro laboral en jóvenes de educación técnico-profesional de la comuna de Victoria". Revista Infancia y educación $\mathrm{N}^{\mathrm{o}} 1$.

VALENZUELA, J. M. (2009). El futuro ya fue. Socioantropología de l@s jóvenes en la modernidad. Tijuana: Colegio de la Frontera.

VERDEJO, J. (s.f.). "Educación permanente y formación de capital humano: El rol de la educación técnica de nivel superior en Chile". Serie Indagación, 29. Disponible en: http://www.expansiva.cl/media/publicaciones/indagacion/documentos/201010041207 $\underline{56 . p d f}$

WELLER, J. (2006). Los jóvenes y el los jóvenes y el empleo en América Latina. Desafíos y perspectivas ante el nuevo escenario laboral. Colombia: CEPAL/Mayol Ediciones. 